\title{
openheart Tip of the iceberg: a tertiary care centre retrospective study of left ventricular hypertrophy aetiologies
}

\author{
Maxime Beneyto (10 ,1,2 Eve Cariou, ${ }^{1,2}$ Jérémy Brunel, ${ }^{1,2}$ Alex Scripcariu, ${ }^{1,2}$ \\ Hubert Delasnerie, ${ }^{1,2}$ Stéphanie Brun, ${ }^{1,2}$ Yoan Lavie-Badie (D) ,1,2,3 \\ Delphine Dupin Deguine, ${ }^{4}$ Michel Galinier, ${ }^{1,2}$ Didier Carrié (D) , ${ }^{1,2}$ Olivier Lairez ${ }^{1,2,3}$
}

To cite: Beneyto M, Cariou E, Brunel J, et al. Tip of the iceberg: a tertiary care centre retrospective study of left ventricular hypertrophy aetiologies. Open Heart 2021;8:e001462. doi:10.1136/ openhrt-2020-001462

Received 23 September 2020 Revised 16 November 2020 Accepted 1 December 2020

Check for updates

(c) Author(s) (or their employer(s)) 2021. Re-use permitted under CC BY-NC. No commercial re-use. See rights and permissions. Published by BMJ.

${ }^{1}$ Cardiology, CHU Toulouse Pôle Cardiovasculaire et Métabolique Toulouse, France

${ }^{2}$ Cardiac Imaging Centre, CHU Toulouse Pôle Cardiovasculaire et Métabolique, Toulouse, France ${ }^{3}$ Nuclear Medicine, CHU Toulouse Département de Médecine Nucléaire, Toulouse, France

${ }^{4}$ Genetics, CHU Toulouse, Toulouse, France

Correspondence to Dr Maxime Beneyto; beneyto. maxime@gmail.com

\section{ABSTRACT}

Aims To phenotype patients referred to a tertiary centre for the exploration of a left ventricular hypertrophy (LVH) starting from $12 \mathrm{~mm}$ of left ventricular wall thickness (LVWT).

Methods and results Consecutive patients referred for aetiological workup of LVH, beginning at $12 \mathrm{~mm}$ of LVWT were retrospectively included in this tertiary single-centred observational study. Patients presenting with severe aortic stenosis were excluded. Aetiological workup was reviewed for each subject and aetiologies were adjudicated by expert consensus.

Among 591 patients referred for LVH aetiological workup, $41 \%$ had a maximal LVWT below $15 \mathrm{~mm}$. LVH aetiologies were led by cardiac amyloidosis (CA, 34.3\%), followed by sarcomeric hypertrophic cardiomyopathy (S-HCM, $32.1 \%)$, hypertensive cardiomyopathy (21.7\%), unknown aetiology (7.6\%) and other (4.2\%), including AndersonFabry's disease (1.7\%). CA and S-HCM affected over 50\% of patients with mild LVH (12-14 mm); the prevalence of these aetiologies rose with LVH severity. Among patients with Anderson-Fabry's disease, $4(40 \%)$ had a maximal LVWT $<15 \mathrm{~mm}$.

Conclusions Mild LVH (ie, 12-14 mm) conceals multiple aetiologies that can lead to specific treatment, cascade family screening and specific follow-up. Overall, CA is nowadays the leading cause of LVH in tertiary centers.

\section{INTRODUCTION}

Current 2014 guidelines for the diagnosis and management of hypertrophic cardiomyopathies (HCMs) from the European Society of Cardiology ${ }^{1}$ define HCM in adults as 'a wall thickness (WT) $\geq 15 \mathrm{~mm}$ in one or more left ventricular (LV) myocardial segments-as measured by any imaging technique (echocardiography, cardiac magnetic resonance imaging (CMR) or CT) - that is not explained solely by loading conditions'. This threshold is set on the basis of historical studies ${ }^{2}$ in the field of HCMs in which an arbitrary value of $15 \mathrm{~mm}$ was used. However, there is no scientific rationale for this cut-off

\section{Key questions}

What is already known about this subject?

- Current European guidelines define hypertrophic cardiomyopathy (HCM) as an increased left ventricular wall thickness (LVWT) $\geq 15 \mathrm{~mm}$ that is not explained by loading conditions.

- This thickness cutoff is arbitrary and solely based on the methods of historical studies on HCM.

- The admitted distribution of HCM aetiologies mainly relies on genetic studies and is about $60 \%$ sarcomere gene mutation, $30 \%$ unknown and $10 \%$ other.

What does this study add?

- Many patients exhibit only mild left ventricular hypertrophy (LVH) (ie, LVWT 12-14 mm).

- Multiple meaningful LVH aetiologies are concealed in this group.

- Overall, amyloidosis is an increasing cause of LVH.

How might this impact on clinical practice?

- The definition of HCM could be revised.

- The threshold to initiate explorations could be lowered to allow early detection, especially considering the emergence of specific treatments for common LVH aetiologies.

value. Studies carried out in healthy subjects have shown that the normal LVWT range is 6-11 mm.

The admitted distribution of HCM aetiologies (relying on various, mostly genetic-based, studies) is presented as such: $40 \%-60 \%$ sarcomeric protein gene mutations, 25\%-30\% unknown and 5\%-10\% genetic and non-genetic causes. ${ }^{1}$ This last category aggregates numerous and diverse aetiologies: inborn errors of metabolism, neuromuscular diseases, mitochondrial diseases, malformation syndromes, amyloidosis, newborn of diabetic mother and drug-induced HCMs.

Clinical practice challenges these statements. First, there seem to exist many HCM diagnoses below the $15 \mathrm{~mm}$ cut-off. Second, 
real-life distribution of HCM aetiologies seems to diverge from the one of the guidelines.

The aim of this study was to phenotype patients referred to a tertiary centre for the exploration of a $\mathrm{LV}$ hypertrophy (LVH) starting from $12 \mathrm{~mm}$ of LVWT.

\section{METHODS}

\section{Study population and data collection}

The medical records of all consecutive patients referred to our tertiary University Hospital, Department of cardiac investigations, Toulouse, France, for the diagnostic work-up of LVH were retrospectively reviewed from January 2015 to July 2019. To accurately describe this population, a lower LVWT cut-off value than the one defined in the upmentioned guidelines was used and all subjects with a maximal LVWT $\geq 12 \mathrm{~mm}$ as measured by transthoracic echocardiography (TTE) were included in the study. Patients presenting with severe aortic valve stenosis (AS), bioprosthetic aortic valve stenotic degeneration and obstructive subaortic membranes were excluded. Patients with a definite LVH aetiology have been subsequently divided into three tertiles allowing a three-stage LVH gradation: mild (maximal LVWT 12-14 mm), moderate (maximal LVWT 15-16 mm) and severe (maximal LVWT $\geq 17 \mathrm{~mm}$ ).

Medical records of all patients were comprehensively reviewed to collect clinical, electrocardiographic, laboratory, imaging and clinical pathology data. The workup algorithm used in our centre is presented in figure 1 . Based on these data, we collegially adjudicated the aetiology of each patient's LVH. For the specific diagnosis of hypertensive cardiomyopathy (HTN-CMP), the following criteria were used: (1) elevated blood pressure at two or more distinct timepoints, (2) at least two antihypertensive medications prescribed and (3) exclusion of other LVH aetiologies.

The investigation conforms with the principles outlined in the Declaration of Helsinki. All patients were informed at the admission that their clinical data could be used for research purpose and gave their consent.

\section{TTE review}

All TTEs had been performed on General Electric ultrasound systems (General Electric Healthcare, Boston, Massachusetts, USA). All TTE loops were reviewed by a trained cardiologist to assess LV walls and chamber dimensions and left and right ventricle systolic function using EchoPAC Software v202 R34.0 (General Electric, Boston, Massachusetts, USA). Two-dimensional measurements were performed unless LV alignment allowed M-mode measurements.

\section{Statistical analysis}

Continuous variables were presented as medians with IQR. Categorical variables were expressed as numbers and percentages. Count data were compared using Fisher's exact tests or Pearson's $\chi^{2}$ tests when applicable. A bilateral $\mathrm{p}<0.05$ was considered statistically significant. In post-hoc analyses, $\mathrm{p}$ values were adjusted with Holm's method. All statistical tests were performed using the $\mathrm{R}$ software V.3.6.2 (R Foundation for Statistical Computing, Vienna, Austria).

\section{RESULTS}

Nine hundred and ninety-three patients with LVH were admitted to our department of cardiac investigations 


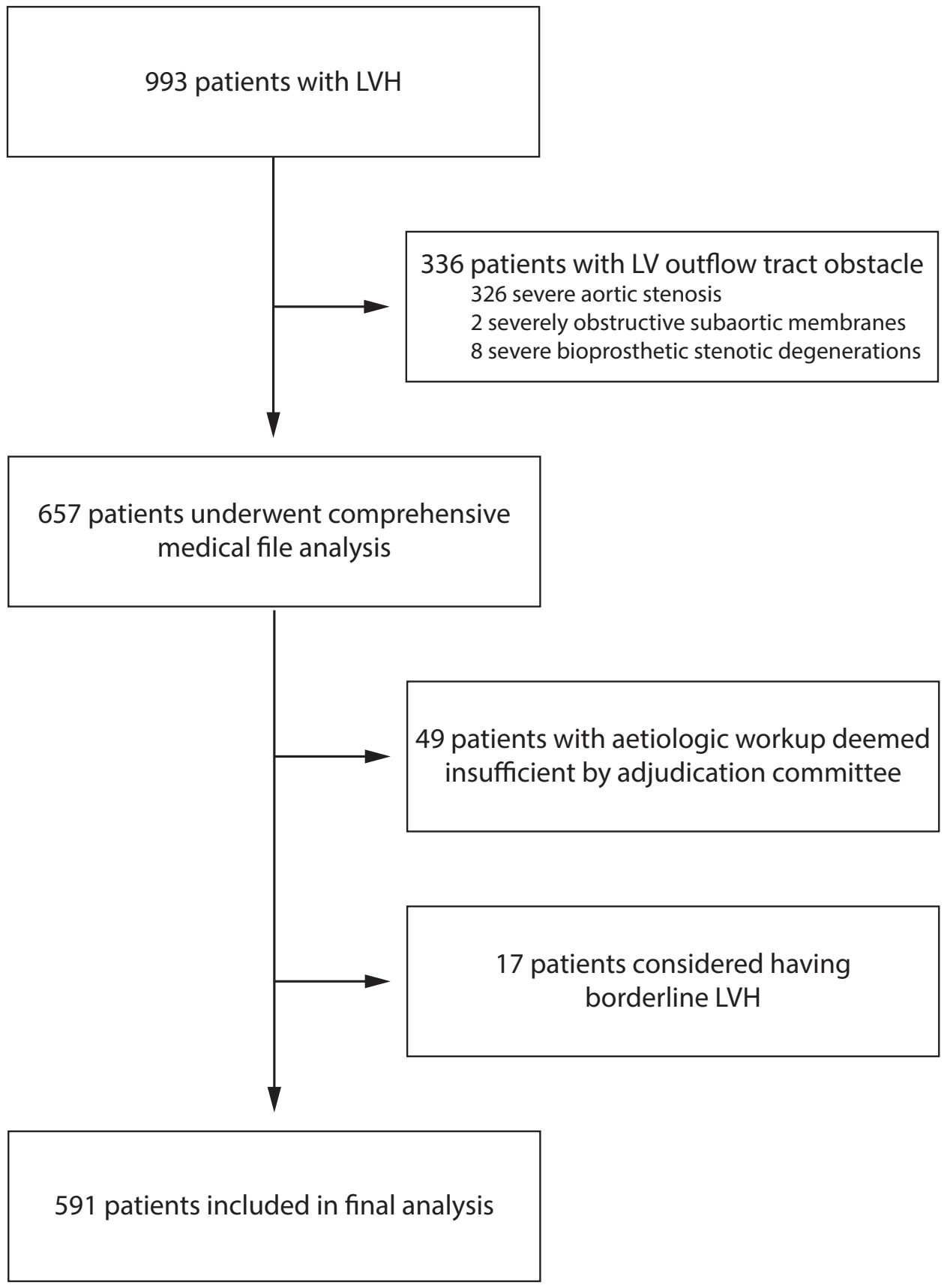

Figure 2 Study flow chart. LV, left ventricle; LVH, left ventricular hypertrophy.

during the studied period. All of them were referred by their cardiologist. Three hundred and twenty-six patients with severe AS were excluded, along with eight patients with bioprosthetic aortic valve stenotic degeneration and two patients with obstructive subaortic membrane. The adjudication committee considered the patient's workup was lacking elements for 49 patients, withholding themselves to reasonably conclude on the LVH aetiology, which led to a posteriori exclusion. Finally, the concomitant occurrence of (1) borderline LVWT by TTE, (2) no LVH with CMR (maximum LVWT $<12 \mathrm{~mm}$ ) and (3) no aetiological lead after a complete workup led to conclude to a TTE false positive and to their withdrawal from the final analysis for 17 patients. The flow chart of the study is presented in figure 2 .

\section{Population characteristics}

Population characteristics are presented in table 1. Briefly, it was mainly composed of mildly symptomatic ageing men presenting with overweight. Arterial hypertension was notably prevalent as it affected 372 subjects (62.9\%); it remained true whatever the LVH aetiology with a prevalence consistently over 50\%. Electrical hypertrophy was common (132 patients, 22.4\%) whereas history of ventricular arrhythmia or implantable cardioverterdefibrillator was rare (40 subjects, 6.8\%). Population 


\begin{tabular}{lc}
\hline Table 1 & \\
\hline Characteristic & \\
\hline Age $(\mathrm{y})$ & $69(56-79)$ \\
\hline Female, N (\%) & $178(30.1)$ \\
\hline BMI $\left(\mathrm{kg} / \mathrm{m}^{2}\right)$ & $25.9(23.1-29.4)$ \\
\hline BSA $\left(\mathrm{m}^{2}\right)$ & $1.85(1.72-1.98)$ \\
\hline Arterial hypertension, N (\%) & $372(62.9)$ \\
\hline Neuromuscular disorder, N (\%) & $117(19.8)$ \\
\hline NYHA class & $2(1-2)$ \\
Electrical hypertrophy, N (\%) & $132(22.4)$ \\
Conduction disorder or PPM, N (\%) & $304(51.5)$ \\
Ventricular arrhythmia or ICD, N (\%) & $40(6.8)$ \\
\hline
\end{tabular}

BMI, body mass index; BSA, body surface area; ICD, implantable cardioverter-defibrillator; NYHA, New York Heart Association; PPM, permanent pacemaker.

characteristics according to $\mathrm{LVH}$ aetiology are detailed in online supplemental material S1.

Main TTE findings are displayed in table 2. Concisely, the median of maximal LVWT was $15 \mathrm{~mm}$, only $67.5 \%$ of patients had increased indexed LV mass, $69.7 \%$ had preserved LV ejection fraction (EF) but merely $16.5 \%$ had normal LV global longitudinal strain $(\leq-18 \%)$, and about $70 \%$ had normal right ventricular longitudinal function whether assessed by tricuspid annular plane systolic excursion or tricuspid annulus $S^{\prime}$ wave velocity. Fourteen per cent of subjects presented with LV outflow tract obstruction with a median maximal gradient of $60 \mathrm{~mm} \mathrm{Hg}$. Strikingly, the most common aetiology in patients presenting with normal indexed LV mass was S-HCM $(41.1 \%)$. Altered LV global longitudinal strain consistently reached about $75 \%-80 \%$ in each aetiology, rising up to $95 \%$ and $92 \%$ in cardiac amyloidosis (CA) and other aetiologies, respectively.

Main TTE findings according to LVH aetiology are detailed in online supplemental material S2.

\begin{tabular}{|c|c|c|}
\hline TTE parameter & Median & IQR \\
\hline Maximal LVWT (mm) & 15 & $13-17$ \\
\hline Indexed LV mass ( $\left.\mathrm{g} / \mathrm{m}^{2}\right)$ & 124 & $102-154$ \\
\hline Indexed LA volume (mL/m²) & 45 & $35-58$ \\
\hline LVEF (\%) & 57 & $45-64$ \\
\hline LV GLS (\%) & -13.4 & -16.6 to -10.1 \\
\hline TAPSE $(\mathrm{mm})$ & 19 & $15-23$ \\
\hline $\mathrm{S}^{\prime} \mathrm{T}(\mathrm{cm} / \mathrm{s})$ & 11.0 & $9.0-14.0$ \\
\hline
\end{tabular}

LA, left atrium; LVEF, left ventricular ejection fraction; LV GLS, left ventricular global longitudinal strain; LVWT, left ventricular wall thickness; TTE, transthoracic echocardiography; S'T, tricuspid annulus S' wave velocity; TAPSE, tricuspid annular plane systolic excursion.

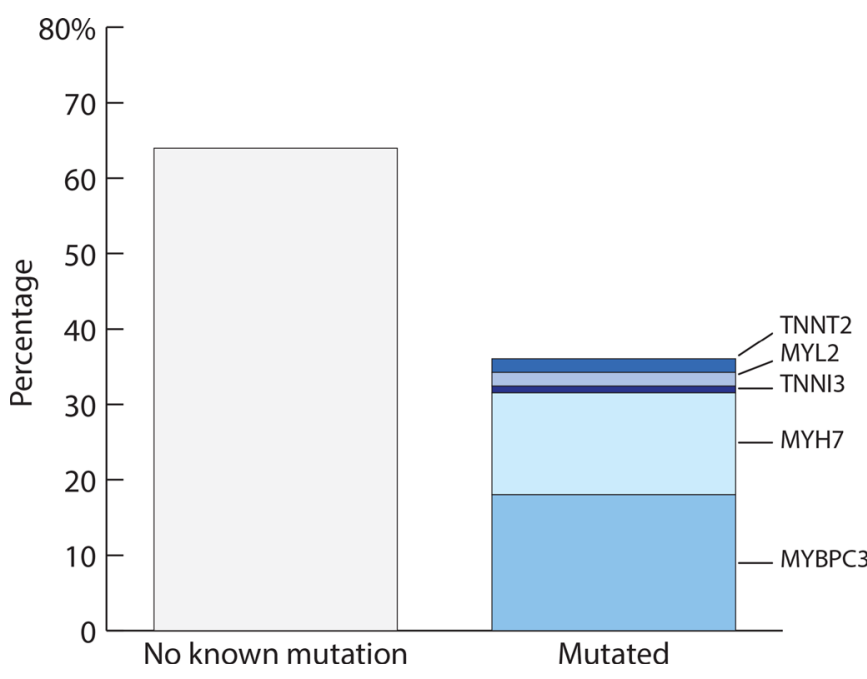

Figure 3 Genetic findings among patients with sarcomeric hypertrophic cardiomyopathy and known genetic status. MYBPC3, myosin binding protein 3; MYH7, myosin heavy chain 3; MYL2, myosin regulatory light chain 2; TNNI3, troponin I 3; TNNT2, troponin T 2.

\section{Distribution of LV aetiologies}

Distribution of LVH aetiologies is presented in online supplemental figure 1. Overall, the most common diagnosis was CA, found in almost a third of patients. The vast majority was transthyretin amyloidosis (ATTR) and especially wild-type ATTR (online supplemental material S3). Among patients with cardiac ATTR, 21.8\% had inherited ATTR (aged 70 (64-73) years). Light-chain (AL) amyloidosis accounted for a fifth of CA cases. Anecdotally, three patients had cardiac involvement of AA amyloidosis and one of apolipoprotein A2 amyloidosis.

Diagnosis of CA was closely followed by S-HCM, which affected more than a quarter of patients. Among those in whom genetic testing had been performed, most had no known mutation (53.0\%; figure 3, online supplemental material S4); otherwise, most common mutated sarcomere genes were MYBPC3 (14.9\%), MYH7 (11.2\%), TNNT2 (1.5\%), MYL2 (1.5\%) and TNNI3 (0.7\%) (genetic analysis was restricted to this set of genes in most patients).

HTN-CMP was the third main aetiology of LVH, it accounted for a fifth of the population.

Twenty-five $(4.2 \%)$ patients had other aetiologies; mostly Anderson-Fabry's disease $(\mathrm{n}=10,1.7 \%)$, but also left ventricular non-compaction (LVNC) of the hypertrophic subtype $(\mathrm{n}=4,0.7 \%)$, drug-induced HCM (tacrolimus $\mathrm{n}=2,0.3 \%$; hydroxychloroquine $\mathrm{n}=1,0.2 \%$ ), mitochondrial cytopathy $(\mathrm{n}=2,0.3 \%)$ and isolated cases $(0.1 \%$ each $)$ of hypereosinophilic syndrome, Noonan syndrome with multiple lentigines, cirrhosis, transient myocardial oedema due to Tako-Tsubo syndrome, postcardiac arrest myocardial oedema and generalised lipodystrophy. LVH aetiology remained undetermined for $7.6 \%$ of the population. 
Table 3 Left ventricular hypertrophy aetiologies detailed according to maximal left ventricular wall thickness

\begin{tabular}{llll}
\hline Aetiology, N (\%) & $\mathbf{1 2 - 1 4 \mathbf { m m }}$ & $\mathbf{1 5 - 1 6 \mathbf { m m }}$ & $\geq \mathbf{1 7} \mathbf{~ m m}$ \\
\hline Amyloidosis & $67(27.9)$ & $60(35.7)$ & $76(41.5)$ \\
Sarcomeric HCM & $63(26.2)$ & $51(30.4)$ & $76(41.5)$ \\
Hypertensive CMP & $71(29.6)$ & $40(23.8)$ & $17(9.3)$ \\
Undetermined & $23(9.6)$ & $11(6.5)$ & $11(6.0)$ \\
Other & $16(6.7)$ & $6(3.6)$ & $3(1.6)$ \\
\hline
\end{tabular}

CMP, cardiomyopathy; HCM, hypertrophic cardiomyopathy.

\section{Population below the European Society of Cardiology LVH threshold}

Two hundred and forty ( $40.6 \%$ ) patients had a maximum LVWT between 12 and $14 \mathrm{~mm}$. Similar results were obtained using CMR measurement. Among them, 217 (90.4\%) had a definite aetiology to their LVH.

Distribution of LVH aetiologies according to the LVH gradation is presented in table 3 (online supplemental figure 1). The distribution of LVH aetiologies significantly differed across the three grades of LVH (overall $\mathrm{p}<1.10^{-5}$; mild vs moderate $\mathrm{p}=0.16$, moderate vs severe $\mathrm{p}<0.01$, mild vs severe $\mathrm{p}<1.10^{-7}$ ). In this subset of the population, LVH aetiologies other than undetermined or HTN-CMP accounted for one half of subjects; undetermined aetiologies were not infrequent. Prevalence of $\mathrm{CA}$ and S-HCM increased with maximum LVWT whereas HTN-CMP, undetermined and other aetiologies decreased.

The three groups of LVH severity only differed on two clinical characteristics: age (higher LVH grades in older patients) and arterial hypertension (less frequent in higher LVH grades). These differences are driven by the increased prevalence of ATTR in more severe LVHs and of HTN-CMP in milder LVHs.

In the subset of patients presenting with $\mathrm{CA}$, the distribution of each subtype of CA differed according to maximum LVWT. The proportion of AL-CA was greater in patients with milder LVH. In patients with ATTR, the proportion of inherited ATTR decreased with increasing maximum LVWT. Other forms of CA were only found in mild LVHs.

Considering subjects presenting with S-HCM, neither the prevalence nor the distribution of sarcomere gene mutations was influenced by LVH severity.

\section{DISCUSSION}

In this study, we described the phenotype of patients referred to our tertiary centre for the aetiological work-up of LVH. The most common aetiology was CA followed by S-HCM and then HTN-CMP. LVH was only mild (LVWT $12-14 \mathrm{~mm}$ ) in $41 \%$ of patients, yet meaningful diagnoses (in terms of prognosis, treatment and follow-up) could be made in more than half of them.

\section{LVH definition}

No reference supports the selection of $15 \mathrm{~mm}$ as the optimal cut-off for HCM diagnosis in the 2014 guidelines. ${ }^{1}$ Moreover, some room is left to perform a complete aetiological work-up for lesser degrees of wall thickening (13-14 mm), which suggests that they might need to be managed just the same as by the definition HCMs. Our study suggests a need in revising this definition: considering only patients with maximum LVWT $\geq 15 \mathrm{~mm}$ would have led to miss 146 (25\%) LVH aetiologies other than HTN-CMP or undetermined. We are well aware that putting this into practice strictly would generate an important increase in healthcare expenses because ageing patients easily tend to present with maximum LVWT $\geq 12 \mathrm{~mm}$. It may be useful to develop easily available 'red flags' (eg, young age, no or controlled arterial hypertension, family history, neuromuscular disorder, abnormal ECG, etc) that would trigger an aetiologic workup. We were confronted ourselves to that issue with some patients presenting borderline LVWT in TTE and a negative yield of the aetiological workup. In these patients, LVWT was not increased in CMR. We believe it is safe to consider them free of LVH (which does not necessarily mean free of heart disease). Maybe the use of the upmentioned red flags would have avoided further investigations.

An absolute cut-off of maximal LVWT to define LVH could in itself be criticised; such a boundary seldom applies indistinctly to all subgroups of a population. It may be interesting to adjust this cut-off to sex, height, race, LV afterload or physical activity (amount and type of exercise). Additionally, an absolute cut-off might overlook relative $\mathrm{LVH}$ in patients presenting with dilated cardiomyopathy.

\section{HCM aetiologies}

Our data suggest that the proportion of S-HCM may be overrepresented in European Society of Cardiology guidelines. A lead to explain this difference is that the studies they are based on have been focusing on a genetic standpoint, which may overlook non-genetic aetiologies, or even non-sarcomeric genetic aetiologies.

In our population, the genetic yield in patients with S-HCM is $36 \%$. It is slightly below the range found by previous studies which extends from $38 \%$ to $53 \%,{ }^{4-7}$ though fewer genes were analysed in most of our patients. Otherwise, we roughly found a similar distribution of the different mutated sarcomeric genes. ${ }^{5-8}$

A recently growing corpus of evidence suggests that prevalence of CA (especially wild-type ATTR) has been greatly underestimated in the past. ${ }^{9}{ }^{10}$ Autopsy series showed $25 \%$ prevalence in the elderly. ${ }^{11}{ }^{12}$ Almost $30 \%$ CA have been found diagnosed in patients with heart failure with preserved EF. ${ }^{13}$ Our study is in line with these recent findings; it is tempting to extrapolate that $\mathrm{CA}$ is at least just as prevalent as S-HCM, that is, 1 in 500 individuals. The up-mentioned underestimation of ATTR frequency also explains the high TTR-to-AL amyloidosis ratio we observed, unlike what have been published before. ${ }^{14}$ 
The exact proportion of inherited ATTR among patients presenting with cardiac ATTR is currently unknown. Two studies estimated this proportion at $12 \%$ and $36 \%$ of patients with cardiac ATTR. ${ }^{15} 16$ Accordingly, in patients who underwent genetic testing, we report $21.8 \%$ of TTR gene mutations, underlining the need of systematic genetic screening in patients with cardiac ATTR, regardless of age.

Genetic and enzymatic assay-based studies have estimated the prevalence of Anderson-Fabry's disease from $0.5 \%$ to $3 \%$ in populations of $\mathrm{HCM}^{8}{ }^{817-20}$ which corresponds to this present study's finding $(1.5 \%)$.

Depending on definition and study population, LVNC prevalence varies from $0.01 \%$ to $3 \% .^{21} 22$ To our knowledge, no prevalence datum exists about the specific hypertrophic subtype of LVNC.

Though a linear correlation exists between the degrees of hypertension and LVH, ${ }^{23}{ }^{24}$ the diagnosis of HTN-CMP remains challenging. The marked prevalence of hypertension across our population advises caution. Yet obviously necessary, history of arterial hypertension cannot be assumed sufficient to conclude to HTN-CMP, especially considering that some aetiologies of HCM (chiefly amyloidosis and Anderson-Fabry's disease) generate renal impairment which can in turn induce hypertension.

\section{Study limitations}

Our study sharing all the limitations and biases associated with retrospective and single-site studies, our population does not reflect the entire panel of patients with LVH. Since the study was set in a tertiary care centre, our population is likely to select more complex cases than would have an ambulatory one, even more so with the exclusion of LV outflow obstructions. In this regard, HTN-CMP in particular may be underrepresented.

Additionally, we focused on patients who were hospitalised at least once in the cardiology wards of our hospital. This could explain why our study lacks neuromuscular disorder-associated HCMs (Friedereich's ataxia, Pompe disease, Danon disease, etc) or other syndromic genetic HCMs (eg, Noonan syndrome), as their general follow-up is performed by other specialists and their cardiovascular evaluation seldom requires hospitalisation in a cardiology department.

Prospective multicentric studies are warranted to lift these limitations and define the most appropriate red flags to avoid unnecessary workups.

\section{CONCLUSION}

LVH begins before the arbitrary LVWT threshold of $15 \mathrm{~mm}$ and there is a wide range of diagnoses from the threshold of $12 \mathrm{~mm}$. The evolution of prevention strategies and treatments according to the different aetiologies should encourage to review explorations thresholds to allow early detection and treatment.

Contributors $\mathrm{EC}$ and $\mathrm{OL}$ participated in the design of the study. $\mathrm{MB}, \mathrm{EC}, \mathrm{JB}, \mathrm{AS}$, $\mathrm{HD}$ and $\mathrm{SB}$ were involved in data collection. MB performed the statistical analysis.
$M B, E C$ and $O L$ interpreted the findings and drafted the manuscript and provided final approval of the version to be published. MB, EC, YL-B, DDD, MG, DC and $\mathrm{OL}$ contributed to the discussion. All authors have read and approved the final manuscript. $\mathrm{OL}$ is responsible for the final content as guarantor.

Funding The authors have not declared a specific grant for this research from any funding agency in the public, commercial or not-for-profit sectors.

Competing interests Olivier Lairez received research support and personal compensation for consulting, serving on a scientific advisory board, speaking or other activities with Alnylam, Amicus, Genzyme, Pfizer and Shire-Takeda. Eve Cariou received personal compensation for speaking with Pfizer.

Patient consent for publication Not required.

Ethics approval Our institutional review board (CHU Rangueil) approved the study and registered it under the reference RnIPH 2020-85.

Provenance and peer review Not commissioned; externally peer reviewed.

Data availability statement Data are available upon reasonable request to the corresponding author.

Open access This is an open access article distributed in accordance with the Creative Commons Attribution Non Commercial (CC BY-NC 4.0) license, which permits others to distribute, remix, adapt, build upon this work non-commercially, and license their derivative works on different terms, provided the original work is properly cited, appropriate credit is given, any changes made indicated, and the use is non-commercial. See: http://creativecommons.org/licenses/by-nc/4.0/.

\section{ORCID iDs}

Maxime Beneyto http://orcid.org/0000-0002-9897-6807

Yoan Lavie-Badie http://orcid.org/0000-0002-8310-4512

Didier Carrié http://orcid.org/0000-0003-2479-8144

\section{REFERENCES}

1 Elliott PM, Anastasakis A, Borger MA. Esc guidelines on diagnosis and management of hypertrophic cardiomyopathy: the task force for the diagnosis and management of hypertrophic cardiomyopathy of the European Society of cardiology (ESC). Eur Heart $J$ 2014;2014:2733-79.

2 Maron BJ, Epstein SE. Hypertrophic cardiomyopathy. Am J Cardiol 1980;45:14:14-154.

3 Kou S, Caballero L, Dulgheru R, et al. Echocardiographic reference ranges for normal cardiac chamber size: results from the NORRE study. Eur Heart J Cardiovasc Imaging 2014;15:680-90.

4 Van Driest SL, Ommen SR, Tajik AJ, et al. Yield of genetic testing in hypertrophic cardiomyopathy. Mayo Clin Proc 2005;80:739-44.

5 Brito D, Miltenberger-Miltenyi G, Vale Pereira S, et al. Sarcomeric hypertrophic cardiomyopathy: genetic profile in a Portuguese population. Rev Port Cardiol 2012;31:577-87.

6 Lopes LR, Zekavati A, Syrris P, et al. Genetic complexity in hypertrophic cardiomyopathy revealed by high-throughput sequencing. J Med Genet 2013;50:228-39.

7 Kassem HS, Azer RS, Saber-Ayad M, et al. Early results of sarcomeric gene screening from the Egyptian national BA-HCM program. J Cardiovasc Transl Res 2013;6:65-80.

8 Coats CJ, Elliott PM. Genetic biomarkers in hypertrophic cardiomyopathy. Biomark Med 2013;7:505-16.

9 Gertz MA, Benson MD, Dyck PJ, et al. Diagnosis, prognosis, and therapy of transthyretin amyloidosis. J Am Coll Cardiol 2015;66:2451-66.

10 Ruberg FL, Grogan M, Hanna M, et al. Transthyretin amyloid cardiomyopathy. J Am Coll Cardiol 2019;73:2872-91.

11 Tanskanen M, Peuralinna T, Polvikoski T, et al. Senile systemic amyloidosis affects $25 \%$ of the very aged and associates with genetic variation in alpha2-macroglobulin and tau: a populationbased autopsy study. Ann Med 2008;40:232-9.

12 Cornwell GG, Murdoch WL, Kyle RA, et al. Frequency and distribution of senile cardiovascular amyloid. Am J Med 1983;75:618-23.

13 Bennani Smires Y, Victor G, Ribes D, et al. Pilot study for left ventricular imaging phenotype of patients over 65 years old with heart failure and preserved ejection fraction: the high prevalence of amyloid cardiomyopathy. Int J Cardiovasc Imaging 2016;32:1403-13.

14 Crotty TB, Li CY, Edwards WD, et al. Amyloidosis and endomyocardial biopsy: correlation of extent and pattern of deposition with amyloid immunophenotype in 100 cases. Cardiovasc Pathol 1995;4:39-42. 
15 Kieninger B, Eriksson M, Kandolf R, et al. Amyloid in endomyocardial biopsies. Virchows Arch 2010;456:523-32.

16 Eriksson M, Büttner J, Todorov T, et al. Prevalence of germline mutations in the TTR gene in a consecutive series of surgical pathology specimens with ATTR amyloid. Am J Surg Pathol 2009;33:58-65.

17 Monserrat L, Gimeno-Blanes JR, Marín F, et al. Prevalence of Fabry disease in a cohort of 508 unrelated patients with hypertrophic cardiomyopathy. J Am Coll Cardiol 2007;50:2399-403.

18 Elliott P, Baker R, Pasquale F, et al. Prevalence of Anderson-Fabry disease in patients with hypertrophic cardiomyopathy: the European Anderson-Fabry disease survey. Heart 2011;97:1957-60.

19 Hagège AA, Caudron $E$, Damy T, et al. Screening patients with hypertrophic cardiomyopathy for Fabry disease using a filter-paper test: the focus study. Heart 2011;97:131-6.
20 Shoichiro N, Toshihiro T, Masato M. An Atypical Variant of Fabry's Disease in Men with Left Ventricular Hypertrophy. N Engl J Med 1995;333:6.

21 Oechslin EN, Attenhofer Jost CH, Rojas JR, et al. Long-Term follow-up of 34 adults with isolated left ventricular noncompaction: a distinct cardiomyopathy with poor prognosis. J Am Coll Cardiol 2000;36:493-500.

22 Towbin JA, Lorts A, Jefferies JL. Left ventricular non-compaction cardiomyopathy. The Lancet 2015;386:813-25.

23 Gradman AH, Alfayoumi F. From left ventricular hypertrophy to congestive heart failure: management of hypertensive heart disease. Prog Cardiovasc Dis 2006;48:326-41.

24 Cramariuc D, Gerdts E. Epidemiology of left ventricular hypertrophy in hypertension: implications for the clinic. Expert Rev Cardiovasc Ther 2016;14:915-26. 